\title{
The Gerstmann syndrome in Alzheimer's disease
}

\section{E M Wingard, A M Barrett, G P Crucian, L Doty, K M Heilman}

J Neurol Neurosurg Psychiatry 2002;72:403-405

Background: It remains unclear from lesion studies whether the four signs of the Gerstmann syndrome (finger agnosia, acalculia, agraphia, and right-left confusion) cluster because the neuronal nets that mediate these activities have anatomical proximity, or because these four functions share a common network. If there is a common network, with degeneration, as may occur in Alzheimer's disease, each of the signs associated with Gerstmann's syndrome should correlate with the other three signs more closely than they correlate with other cognitive deficits.

Methods: Thirty eight patients with probable Alzheimer's disease were included in a retrospective analysis of neuropsychological functions.

Results: The four Gerstmann's syndrome signs did not cluster together. Finger naming and calculations were not significantly correlated. Right-left knowledge and calculations also did not correlate.

Conclusions: The four cognitive functions impaired in Gerstmann's syndrome do not share a common neuronal network, and their co-occurrence with dominant parietal lobe injuries may be related to the anatomical proximity of the different networks mediating these functions.

t $\mathrm{n}$ a series of three reports, Josef Gerstmann ${ }^{1-3}$ posited that the combination of finger agnosia, right-left confusion, acalculia, and agraphia constituted a syndrome induced by lesions of the left hemisphere's angular gyrus.

Since Gerstmann's reports there has been controversy about this syndrome. ${ }^{4}$ This controversy has three major components: Firstly, are these disorders an autonomous combination or do these four signs co-occur with other behavioural disorders? Secondly, does the presence of these four signs indicate a lesion in the angular gyrus of the left hemisphere? Thirdly, is there a basic underlying deficit (Grundstorung) that can account for these signs?

Regarding the first and second questions, there have been several studies showing that patients with the four elements of Gerstmann's syndrome also had other neuro-behavioural disorders and that Gerstmann's syndrome is usually associated with large left hemispheric lesions. ${ }^{5}$ More recently, however, there have been reports of patients with discrete anatomical lesions, or functional disruption by electrical stimulation of the angular gyrus, who demonstrated Gerstmann's syndrome and no other neurobehavioural disorders. ${ }^{7-10}$

The findings that Gerstmann's syndrome can be an autonomous combination and is associated with dysfunction of the angular gyrus suggests that there is a basic underlying deficit that can account for these four signs, but what is the underlying nature of these deficits? One possibility is that there are multiple neuronal networks that mediate these separate cognitive activities but that these networks have anatomical proximity. Alternatively, it is possible that a single neuronal network is responsible for mediating, at least in part, all four cognitive skills. Gerstmann ${ }^{3}$ postulated that knowl- edge of both fingers and right/left orientation are supported by knowledge of the body (a shared network for body schema). Critchley ${ }^{11}$ proposed that counting and calculating are first learned on the fingers. Thus, finger knowledge and calculation ability may be supported by a shared neuronal network.

To address the question of whether Gerstmann's syndrome reflects neuroanatomical proximity of independent networks or a common neuronal network, we studied a group of patients with probable Alzheimer's disease who often have difficulties with writing, finger naming, calculations, and right-left orientation. By examining the co-occurrence of the Gerstmann's syndrome components in such patients, we wished to learn whether these four deficits are highly intercorrelated. If the symptoms comprising Gerstmann's syndrome are mediated by a single or shared neuronal network, we would expect that the statistical analysis would show them to cluster. We also predicted that if Gerstmann's syndrome indicates degradation of a single or shared neuronal network, the symptoms would correlate more highly with each other than with other parietal lobe and hippocampal/ basal forebrain cognitive deficits that occur in probable Alzheimer's disease.

\section{METHODS}

\section{Patients}

We reviewed 71 charts of patients with the diagnosis of probable Alzheimer's disease by NINCDS-ADRA criteria. ${ }^{12}$ Patients were excluded if they had structural brain pathology (other than atrophy), depression with electroconvulsive therapy, seizures, associated vascular dementia, or parkinsonism. Depression alone was not an exclusionary criterion. To be included patients had to understand the commands on the mini mental status examination. Thirty eight patients met inclusion criteria, comprising 27 women, 11 men, 29 right handers, one left hander, and eight unrecorded; mean age 71.39 years (range 44-89); mean education 12.48 years (range 7-18); mean duration of symptoms 3.35 years (range $1-10$ ).

\section{Procedure}

All patients with the diagnosis of probable Alzheimer's disease received neuropsychological testing. The mean mini mental status $^{13}$ score was 15.03 (SD 6.57). The mean score on the Boston naming test ${ }^{14}$ was 33.68 (SD 13.69) and the mean score on the Hopkins verbal learning test ${ }^{15}$ was 8.71 (SD 4.48).

All scores for right-left orientation (RL), finger naming (FN), serial seven calculations (CALC), sentence writing (WRIT), praxis (PRAX), map orientation (MAP), forward digit span (DIGIT), and sentence repetition (REP) were scored on a five point scale, and converted to $\mathrm{Z}$ scores based on normative

Abbreviations: RL, right-left orientation; $F N$, finger naming; CALC, serial seven calculations; WRIT, sentence writing; PRAX, praxis; MAP map orientation; DIGIT, forward digit span; HVLT, verbal memory; BNT, confrontational naming 
Table 1 Z scores and values for Pearson's product moment coefficient

\begin{tabular}{|c|c|c|c|c|c|c|c|c|c|c|}
\hline \multicolumn{2}{|c|}{ Test performance } & \multicolumn{9}{|c|}{ Pearson's r } \\
\hline & $\begin{array}{l}\text { Z score mean } \\
\text { (SD) }\end{array}$ & RL & CALC & WRIT & BNT & HVLT & PRAX & MAP & DIGIT & REP \\
\hline $\mathrm{FN}$ & $-3.19(3.31)$ & $0.372^{*}$ & 0.243 & $0.528 * *$ & 0.046 & 0.288 & 0.121 & $0.469 * *$ & $0.345^{*}$ & 0.324 \\
\hline RL & $-3.04(3.27)$ & & 0.213 & $0.432^{*}$ & -0.120 & 0.067 & 0.316 & $0.418^{*}$ & $0.442 * *$ & 0.322 \\
\hline CALC & -1.28 (1.01) & & & $0.383^{*}$ & 0.134 & 0.356 & $0.486^{* *}$ & $0.469 * *$ & $0.528^{* *}$ & 0.337 \\
\hline WRIT & $-0.94(1.63)$ & & & & -0.138 & $0.433^{*}$ & 0.246 & 0.276 & 0.729 ** & $0.394 *$ \\
\hline BNT & $-2.65(1.87)$ & & & & & $0.483^{*}$ & $0.385 *$ & 0.305 & -0.023 & 0.257 \\
\hline HVLT & $-3.16(0.81)$ & & & & & & 0.002 & 0.256 & 0.360 & 0.112 \\
\hline PRAX & $-1.25(2.05)$ & & & & & & & 0.117 & 0.207 & 0.100 \\
\hline MAP & $-1.01(1.60)$ & & & & & & & & $0.359 *$ & $0.563^{* *}$ \\
\hline DIGIT & $-0.13(1.14)$ & & & & & & & & & $0.494^{* *}$ \\
\hline REP & $0.19(2.00)$ & & & & & & & & & 1.000 \\
\hline
\end{tabular}

${ }^{*} p, 0.05 ;{ }^{*} p<0.01$ (two tailed).

FN, finger naming; RL, right-left orientation; CALC, calculations (serial sevens); WRIT, sentence writing; BNT, Boston naming test ${ }^{18}$; HVLT, Hopkins verbal learning test ${ }^{15}$; PRAX, praxis; MAP, map orientation; DIGIT, forward digit span; REP, sentence repetition.

data. ${ }^{13-16}$ To determine how closely the symptoms of Gerstmann's syndrome were intercorrelated relative to their correlation with other parietal and other cognitive deficits, Pearson's product-moment coefficient $(r)$ was calculated for each intervariable relation and a hierarchical cluster analysis was performed.

\section{RESULTS}

$\mathrm{Z}$ scores and values for Pearson's product-moment coefficient are presented in the table for RL, FN, CALC, WRIT, PRAX, MAP, DIGIT, REP, HVLT, and BNT. Two components of the Gerstmann's syndrome variables were significantly correlated: right-left orientation and finger naming $(r=0.372, \mathrm{p}=0.028)$. Calculations and finger naming, however, were not significantly correlated $(r=0.243, \mathrm{p}=0.188)$. Writing was significantly correlated with all of the other components of Gerstmann's syndrome (WRIT-FN: $r=0.528, \mathrm{p}=0.001$; WRITRL: $r=0.432, \mathrm{p}=0.012$; WRIT-CALC: $r=0.383, \mathrm{p}=0.033$ ).

Hierarchical cluster analysis was performed to further delineate the structure of the variable intercorrelations. Scores for each of the variables for the 38 patients were plotted in a geometric space according to their average within group linkage. The hierarchy resulting from clustering the data from the 38 patients is shown in the figure. On this dendrogram, closely

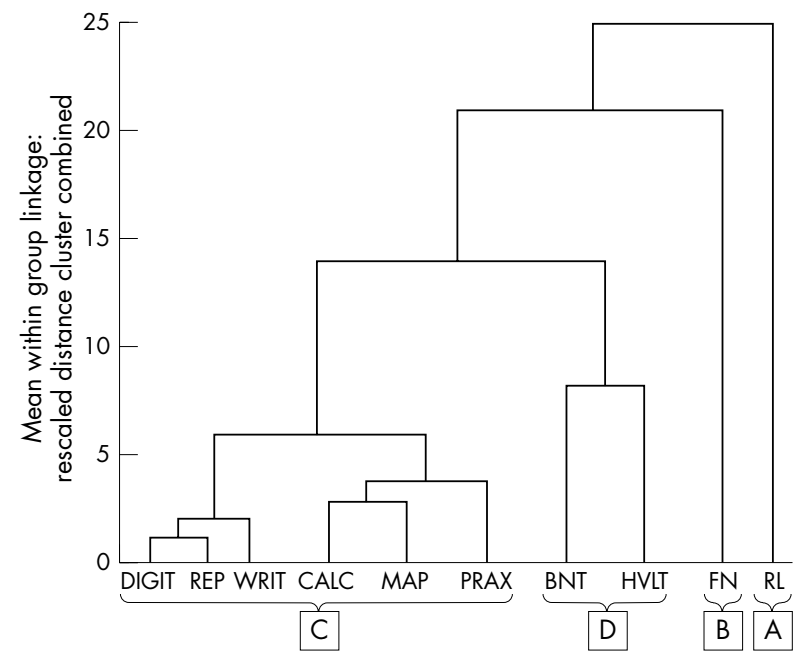

Figure 1 Closely intercorrelated variables are joined at an early stage in the analysis (bottom of the dendrogram) and less closely intercorrelated variables are joined at a later stage in the analysis (top of the dendrogram). A four cluster solution results in clusters A-D $(A=R L ; B=F N ; C=D I G I T, R E P, W R I T, C A L C, M A P$, and PRAX; $D=B N T$ and HVLT). intercorrelated variables are joined at an early stage in the analysis (at the bottom of the dendrogram), and less closely intercorrelated variables are joined at later stages (the top of the dendrogram). Four clusters were recognised in the analysis, identified by marked discontinuity or a "step" in the hierarchy. Although RL and FN were correlated, they did not cluster together, nor did they cluster with WRIT and CALC, the remaining deficits of Gerstmann's syndrome. A four cluster solution results in clusters A-D, with A containing RL, B containing FN, and a third cluster, C, containing WRIT and CALC clustered with other parietal cognitive functions (MAP and PRAX). It should be noted that similar results were found with a second hierarchical cluster analysis using complete (furthest neighbour) linkage.

None of the symptoms of Gerstmann's syndrome clustered with verbal memory (HVLT) or naming (BNT); these two variables formed a fourth cluster, D. These two deficits were also strongly intercorrelated $(r=0.483, \mathrm{p}=0.020)$. We also found that DIGIT was strongly correlated with many cognitive functions (DIGIT-FN: $r=0.345, \mathrm{p}=0.042 ;$ DIGIT-RL: $r=0.442$, $\mathrm{p}=0.008 ; \quad$ DIGIT-CALC: $r=0.528, \mathrm{p}=0.002 ; \quad$ DIGIT-WRIT: $r=0.729, \mathrm{p}<0.001 ;$ DIGIT-MAP: $r=0.359, \mathrm{p}=0.032)$.

\section{DISCUSSION}

By studying Gerstmann's syndrome in patients with degenerative disease rather than in patients with stroke, we hoped to determine whether the four signs of Gerstmann's syndrome were mediated by a common or by different neuronal networks that are in close anatomical proximity. If these four functions share a common network, with degeneration of this network, the four deficits of Gerstmann's syndrome should correlate with each other more closely than with other cognitive signs associated with probable Alzheimer's disease or parietal lobe dysfunction. We found that the four signs of Gerstmann's syndrome do not cluster as a distinct syndrome in probable Alzheimer's disease. Whereas Critchley ${ }^{11}$ suggested that finger knowledge and calculations were closely related, we found no correlation between these functions, suggesting that they did not share a common network. Right-left knowledge also did not correlate with calculation, but functions such as praxis and map finding that are not part of Gerstmann's syndrome did strongly correlate with calculation. Overall, these results suggest that there is not a common neuronal network shared by the four cognitive functions impaired with Gerstmann's syndrome and that the co-occurrence found with discrete lesions may be related to anatomical proximity. We did, however, find a statistically significant correlation between finger naming and right-left orientation that may indicate a shared neuronal network for body schema as proposed by Gerstmann. ${ }^{3}$ 
We found that sentence writing (WRIT) did not correlate with confrontational naming (BNT), but did correlate with the other three functions of Gerstmann's syndrome. Although these findings suggest that the writing deficit was not induced by language deficit, it is unclear how writing is related to other functions of Gerstmann's syndrome. The strong correlation between verbal memory (HVLT) and naming (BNT) suggests that language skills may be important for memory encoding, that degeneration of the basal forebrain may impair both skills, or that there is parallel degradation of two independent networks. The correlation between digit span (DIGIT) and many of the other measured variables was unexpected and may indicate that an adequate span of attention or working memory is necessary for the performance of many cognitive tasks.

\section{ACKNOWLEDGEMENTS}

This work was supported by the National Institutes of Health and the State of Florida Department of Elder Affairs. It was presented to the 5lst annual meeting of the American Academy of Neurology, Toronto, Ontario, April 1999 and published in abstract form in Neurology 1999;52 (suppl 2):AI54.

\section{Authors' affiliations}

E M Wingard, Division of Neurology, Department of Medicine, University of Massachusetts, USA

A M Barrett, Department of Neurology, Penn State College of Medicine, USA

G P Crucian, L Doty, K M Heilman, Department of Neurology, University of Florida College of Medicine, Gainesville, FL32610, USA K M Heilman, Neurology Service, Veterans Affairs Medical Center, Gainesville, FL, USA

Correspondence to: Dr K M Heilman, PO Box 100236, Gainesville, FL32610-0236, USA; heilman@medicine.ufl.edu
Received 8 May 2001

In revised form 20 August 2001

Accepted 5 November 2001

\section{REFERENCES}

1 Gerstmann J. Fingeragnosie: eine umschriebene Stbrung der Orientierung am eigenen Korper. Wien Klin Wochenschr 1924:37:1010-12.

2 Gerstmann J. Fingeragnosie und insolierte Agraphie: ein neues Syndrom. Z ges Neurol Psychiat 1927; 108:152-77.

3 Gerstmann J. Zur Symptomatologie der H-lirniasionen im Obergangsgebiet der unteren Parietal-md mittleren Occipitalwindung Nervenarzt 1930;3:691-5.

4 Benton AL. Gerstmann's syndrome. Arch Neurol 1992;49:445-7.

5 Benton AL. The fiction of the Gerstmann syndrome. J Neurol Neurosurg Psychiatry 1961;24:176-81.

6 Heimburger RF, Demyer W, Reitan RM. Implications of Gerstmann's syndrome. J Neurol Neurosurg Psychiatry 1964;27:52-7.

7 Roeltgen DP, Sevush S, Heilman KM. Pure Gerstmann's syndrome from a focal lesion. Arch Neurol 1983;40:46-7.

8 Varney NR. Gerstmann syndrome without aphasia: a longitudinal study Brain Cogn 1984;3:1

9 Mazzoni M, Pardossi L, Cantini R, et al. Gerstmann syndrome: a case report. Cortex 1990;26:459-67.

10 Morris HH, Luders $\mathrm{H}$, Lesser RP, et al. Transient neuropsychological abnormalities (including Gerstmann's syndrome) during cortical stimulation. Neurology 1984;34:877-83

11 Critchley.

12 McKhann G, Drachman D, Folstein M, et al. Clinical diagnosis of Alzheimer's disease: report of the NINCDS-ADRDA work group under the auspices of Department of Health and Human Services task force on auspices of Department of Health and Human Services

13 Folstein MF, Folstein SE, McHugh PR. "Mini mental state:" a practical method for grading the cognitive state of patients for the clinician. $J$ Psychiatr Res 1975;12:189-98.

14 Kaplan EF, Goodglass H, Weintraub S. The Boston naming test, 2nd ed. Philadelphia: Lea and Febinger, 1983.

15 Brandt J. The Hopkins verbal learning test: development of a new memory test with six equivalent forms. Clin Neuropsychol 1991; 5: 125-42.

16 Doty L, Bowers D, Heilman KM. Florida mental status exam for progressive dementia screening. Presented at the Gerontological Society of America, Boston, MA [abstract]. Gerontologist 1990;30/special issue): $20 \mathrm{~A}$. 